\title{
SIMULATION OF PRESSURE-INDUCED CAVITY DEFORMATION - THE 18SIB04 QUANTUMPASCAL EMPIR PROJECT
}

\author{
J. Zakrisson ${ }^{1}$, I. Silander ${ }^{1}$, C. Forssén ${ }^{1}$, Z. Silvestri ${ }^{2}$, D. Mari ${ }^{3}$, S. Pasqualin ${ }^{3}$, A. Kussicke ${ }^{4}$, P. Asbahr ${ }^{4}$, \\ T. Rubin ${ }^{4}$ O. Axner ${ }^{1}$ \\ ${ }^{1}$ Department of Physics, Umeå University, SE-901 87 Umeå, Sweden, \\ johan.zakrisson@umu.se, isak.silaner@umu.se, clayton.forssen@umu.se, ove.axner@umu.se \\ ${ }^{2}$ LNE-Cnam, 61 rue du Landy, 93120 La Plaine Saint-Denis, France, zaccaria.silvestri@ @nam.fr \\ ${ }^{3}$ INRiM, National Institute of Metrological Research, Strada delle cacce, 91, 10135 Torino, Italy, \\ d.mari@inrim.it, s.pasqualin@inrim.it \\ ${ }^{4}$ Physikalisch-Technische Bundesanstalt (PTB), Abbestr. 2-12, 10587 Berlin, Germany, \\ andre.kussicke@ptb.de, patrick.asbahr@ptb.de, tom.rubin@ptb.de
}

\begin{abstract}
:
The 18SIB04 QuantumPascal EMPIR project aims for development of photon-based standards that can replace primary standards of the SI unit of pressure, the Pascal. In this project, four partners simulated the pressure-induced deformation of a given Fabry-Pérot cavity, using various versions of two types of software, COMSOL Multiphysics ${ }^{\circledR}$ and ANSYS Workbench. It was demonstrated that, for a given geometry and set of material parameters, simulations of the deformation could be performed by the various partners with such small discrepancies that methodological mistakes of the simulation procedures will solely contribute to a sub-ppm uncertainty in the assessments of refractivity of $\mathrm{N}_{2}$.
\end{abstract}

Keywords: EMPIR; QuantumPascal; FEM; Pressure; Refractometry; Deformation.

\section{INTRODUCTION}

The current realization of the Pascal is based on piston gauges and liquid manometers [1,2]. Their performance have remained basically unchanged during the last few decades and, especially for pressures below $100 \mathrm{kPa}$, they suffer from practical and environmental limitations [3, 4].

The 18SIB04 QuantumPascal EMPIR project "Towards quantum-based realizations of the Pascal" - aims to develop photon-based standards for pressure to address these limitations [5]. One of the techniques addressed is Fabry-Pérot (FP) cavity (FPC) based refractometry, which is a sensitive technique for assessment of gas refractivity, density, pressure, and flows by the use of laser

\footnotetext{
1 Umeå University (UmU), Sweden; Conservatoire national des arts et métiers (Cnam), France; National Institute of Metrological Research (INRiM), Italy; and
}

technology. By measuring the refractivity, $n-1$, where $n$ is the index of refraction, and the temperature of a gas, the pressure can be calculated by the use of the Lorentz-Lorenz equation and an equation of state.

With the implementation of the refined SIsystem in May 2019, the uncertainty in the Boltzmann constant was eliminated [6]. This promises primary measurements limited only by the uncertainty in quantum calculations of molar polarizabilities and some virial coefficients, and in the assessment of axial pressure-induced cavity deformation and gas temperature. This means that, in the long term, such primary standards could provide fast and accurate pressure measurements at a fraction of the present cost.

One means to assess the cavity deformation is to simulate it by the use of finite element methods (FEM) [7]. To eliminate the risk of methodological errors in the set-up of the cavity and the execution of the simulation, the first activity (A1.1.1) in the aforementioned project was devoted to verifying that the simulation procedures used by the four partners that address the pressure induced cavity deformation in FPC-based refractometry ${ }^{1}$, here anonymized as partners $\mathrm{A}, \mathrm{B}, \mathrm{C}$, and $\mathrm{D}$, were appropriate. This was done by corroborating that they all could predict the same axial pressureinduced cavity deformation of a given FPC (provided by one of the partners) with a given set of material parameters.

Note that the activity presented in this work was not devoted to an assessment of to which extent the simulated deformation agrees with respect to a previously performed experimental assessment.

Physikalisch-Technische Bundesanstalt

(PTB), 
Instead, using the verified modelling procedure, each partner will, in a subsequent project activity (A1.1.2), model the deformation of a FPC they have access to in their own laboratory, whose deformation they also will experimentally characterize (A1.1.3). Since all partners have FPCs with dissimilar geometries (sizes and spacer materials) and mirror mountings, the concept of accuracy will, by this procedure, at a later stage of the project, be scrutinized from a broad point of view.

It was agreed on that the work should be performed in the following way: after a first round of simulations, performed individually by each of the four partners, the results were to be compared. If discrepancies appeared, their causes were to be identified and resolved. This was to be continued until no significant inconsistencies remained. When consensus regarding the pressure-induced cavity deformation had been reached, it was considered that the set-ups of the cavities and the executions of the simulations did not incorporate any methodological inconsistencies.

\section{DESCRIPTION OF THE CAVITY}

The cavity considered was a previously constructed and characterized cavity from Cnam [8]. The spacer, made of Zerodur ${ }^{\circledR}$, schematically illustrated in Figure 1, is $100 \mathrm{~mm}$ long with an inner and outer diameter of 34 and $56 \mathrm{~mm}$, respectively. On each side, $15 \mathrm{~mm}$ thick mirrors, made of fused silica, with a diameter of $50 \mathrm{~mm}$, are mounted to the cavity spacer by optical contacting.

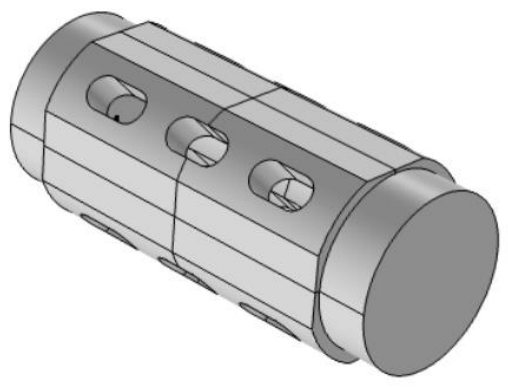

Figure 1: A 3D model of the simulated FP cavity.

To simplify the modelling, the cavity was considered to have flat mirrors. It was agreed on that the material parameters used in the simulations should be those presented in Table 1.

Table 1. Material properties used by all partners in the simulations.

\begin{tabular}{|c|c|c|}
\hline Entity & $\begin{array}{c}\text { Spacer } \\
\text { material } \\
\left(\text { Zerodur }^{\circledR}\right) \\
\end{array}$ & $\begin{array}{c}\text { Mirror } \\
\text { substrate } \\
\text { (fused silica) }\end{array}$ \\
\hline Young's modulus (GPa) & 90.3 & 73 \\
\hline Poisson ratio & 0.24 & 0.155 \\
\hline Density $\left(\mathrm{kg} / \mathrm{m}^{3}\right)$ & 2530 & 2195 \\
\hline
\end{tabular}

\section{SIMULATION PREOCEDURE}

\subsection{Software used}

To ensure that the simulation results were not software dependent the software used in the simulations were, at the start of the project, not only chosen by the partners themselves, it was also emphasised that not all partners should use the same type or version of software. Three partners, A, B, and $\mathrm{D}$, utilized various versions of COMSOL Multiphysics ${ }^{\circledR}$, viz. the versions 5.3a/5.5, 5.4, and 5.5 , respectively, together with the structural mechanics module, while two of the partners, B and C, used ANSYS Workbench - Academic Student 2019 R2 with a static structural toolbox (partner B thus used both types of software).

\subsection{Set-up of the cavity}

To create the 3D model of the cavity the partners $\mathrm{A}$ and $\mathrm{B}$ used the built-in design module in COMSOL Multiphysics ${ }^{\circledR}$. Partner C utilized the DesignModeler Ansys Workbench module to import CAD file created in Autodesk fusion 360. Partner D employed the CAD import module to import 3D models originally created in Autodesk Inventor Professional 2020.

Both the material of the cavity spacer and the mirrors were modelled using a linear-elastic model. The cavity spacer and the mirrors were considered to be unified, which means that they were seen and treated as one joint solid object. To simulate the application of a pressure onto the cavity, a boundary load was applied to all external surfaces.

To decrease computational work, the partners B, $\mathrm{C}$, and $\mathrm{D}$, utilized (three) symmetry planes and simulated solely $1 / 8$ of the cavity. By using such planes, any rigid body motion of the cavity could automatically be eliminated, which facilitated interpretation of the results. The partner that simulated the full cavity, A, used Prescribed Displacement (or Roller) constraints in the symmetry planes to restrict any rigid body movement of the cavity.

Partner A performed (a set of) simulations of the cavity deformation based on modelling of the (entire) cavity using $1 \times 10^{4}$ to $1.7 \times 10^{6}$ mesh elements. Among the partners that simulated $1 / 8$ of the cavity, to describe the modelled part of the cavity, partner B used $1.9 \times 10^{5}$ mesh elements in COMSOL Multiphysics ${ }^{\circledR}$ and $1.7 \times 10^{2}$ to $2.9 \times 10^{6}$ mesh elements in ANSYS Workbench, partner C utilized $3.6 \times 10^{2}$ to $2.1 \times 10^{4}$ elements, while partner D employed $1.2 \times 10^{4}$ to $3.2 \times 10^{6}$ elements. The relevant meshing parameters for the finest mesh used in the simulation by each partner and for each type of software are summarized in Table 2. 
Table 2. Meshing parameters for the finest mesh used in the simulation by each partner.

\begin{tabular}{|l|c|c|c|c|c|}
\hline \multicolumn{1}{|c|}{ Partner } & A - Comsol & B - Comsol & B - Ansys & C - Ansys & D - Comsol \\
\hline Number of mesh elements & 1735324 & 190000 & 2948108 & 21061 & 3168777 \\
\hline Maximum element size (m) & $1.2 \times 10^{-3}$ & $1.3 \times 10^{-3}$ & $1 \times 10^{-3}$ & $2.3 \times 10^{-3}$ & $5 \times 10^{-4}$ \\
\hline Minimum element size (m) & $9 \times 10^{-6}$ & $1.3 \times 10^{-5}$ & $2 \times 10^{-4}$ & $2.8 \times 10^{-4}$ & $5 \times 10^{-6}$ \\
\hline Growth rate & 1.2 & 1.3 & 1.2 & 1.2 & 1.2 \\
\hline Curvature factor & 0.25 & 0.2 & & 0.27 & 0.2 \\
\hline
\end{tabular}

An example of the meshing of the cavity (provided by partner D) is presented in Figure 2.

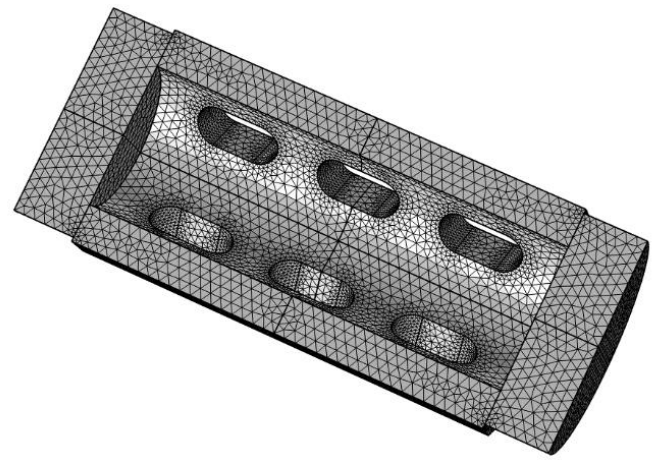

Figure 2. An example of a meshing of the cavity shown in a cross-sectional view.

\section{RESULTS}

\subsection{Typical strain in a pressurized cavity}

A typical pressure-induced deformation of the cavity addressed, created by partner $\mathrm{D}$, is presented in Figure 3. The colours on the cross section surface represent the pressure-normalized longitudinal repositioning of the cavity material (along the optical axis) relative to the centre of the cavity, i.e., $\delta L / P$, where $\delta L$ is the axial repositioning relative the centre of the cavity and $P$ is the pressure.

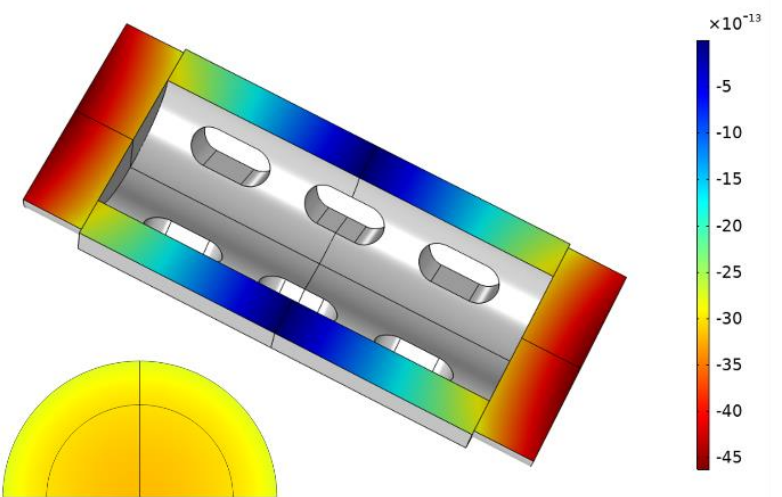

Figure 3. Cross section of the cavity. The colour on the cross section surface represents pressure-normalized longitudinal repositioning of the cavity material relative to the centre of the cavity, $\delta L / P$, in units of $\mathrm{m} \mathrm{Pa}^{-1}$. The semi-disc in the lower left corner represents the same entity for half of the reflective surface of one of the mirrors.

This figure shows that the longitudinal deformation of the cavity is dominated by a (close-to- linear) deformation (compression) of the cavity spacer and that the bending of the mirrors plays a minor role.

The simulations of the cavity deformation were then quantized in terms of a pressure-normalized strain of the cavity, i.e. the relative pressureinduced change in length of the cavity for a given pressure, defined as $(\Delta L / L) / P$, where $\Delta L$ is the change in length between the centre points of the reflective surface of the two mirrors induced by the presence of gas and $L$ is the length of the undeformed cavity (i.e., for $P=0 \mathrm{~Pa}$ ).

\subsection{First round of simulations}

The first round of simulations, based on independent work by each partner, predicted a pressure-normalized strain ranging from $-5.4 \times 10^{-}$ ${ }^{12}$ to $-7.85 \times 10^{-12} \mathrm{~Pa}^{-1}$, with a mean of $-6.49 \times 10^{-12}$ $\mathrm{Pa}^{-1}$ and a $\left(2 \sigma_{\bar{x}}\right)$ confidence interval of $\pm 13 \%$.

This spread was significantly larger than considered adequate. A scrutiny revealed that it was attributed to methodological errors in the setup of the cavity and the execution of the simulations. This led to a revision process in which all partners independently reviewed, in detail, their set-up and execution processes. During this process, the causes for the large spread in results were successively identified. In all cases, they were found to originate from incorrect or inappropriately applied input parameters, boundary conditions, or procedures ascribed to misunderstandings about how the pertinent program interpreted or utilized the various input parameters. When the various misunderstandings were identified, they were remedied.

\subsection{Final set of simulations}

In the final set of simulations, when no methodological set-up or execution errors remained, the partners made systematic investigations of the influence of the number of mesh elements on the predicted pressure-induced deformation. Such a study is shown in Figure 4.

It was found that there is a clear dependence of the pressure-induced strain on the number of mesh elements. For partner D, for example, which performed simulations using $1.2 \times 10^{4}$ to $3.2 \times 10^{6}$ mesh elements, the normalized pressure induced 


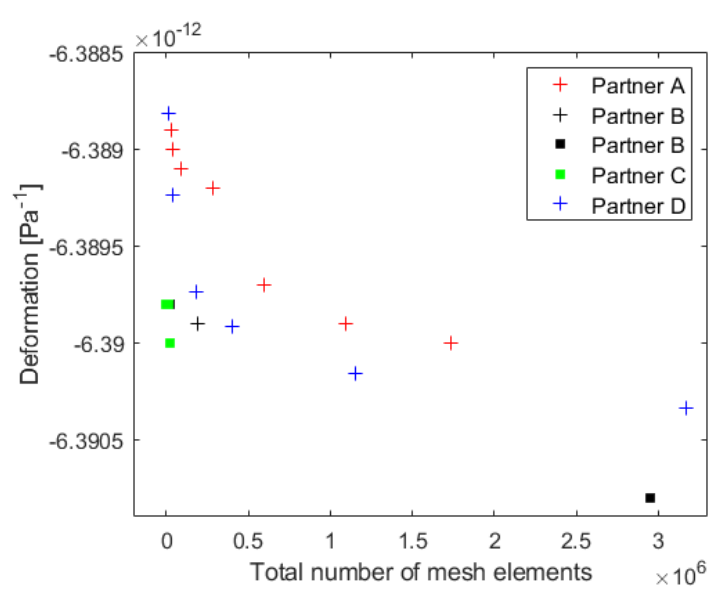

Figure 4. Simulated normalized pressure-induced strain from the four partners. Note that the "plus" makers indicate the use of COMSOL Multiphysics ${ }^{\circledR}$ while the "square" markers denote the use of ANSYS Workbench. Moreover, partner A simulated the entire cavity spacer, while the other partners modelled only $1 / 8$ of it.

deformation ranged from $6.3888 \times 10^{-12}$ to 6.3903 $\times 10^{-12} \mathrm{~Pa}^{-1}$. The simulations performed by the other partners showed a similar behaviour. This implies that it is in general advisory to perform an analysis of the dependence of the strain as a function of the number of mesh elements, and choose an appropriate number of mesh elements for the type of simulation performed.

However, it was also found that the pressureinduced strain levels off asymptotically with the number of mesh elements. This implies that the simulations with the largest number of mesh points are least dependent of the number of mesh points used and are thereby assumed to be those with highest accuracy.

On the other hand, the difference in simulated deformation performed by partner $\mathrm{D}$ when the two largest numbers of mesh points were used (1.2 and $3.2 \times 10^{6}$ elements, respectively) is only $2 \times 10^{-16}$ $\mathrm{Pa}^{-1}$, which, for $\mathrm{N}_{2}$, corresponds to a relative uncertainty in the assessed refractivity of solely $0.075 \mathrm{ppm}$. This implies that simulations with an excessive number of mesh elements may not necessarily be needed

Figure 4 shows that the pressure-induced strain ranged, for each partner (simulated using the largest number of mesh points), from $-6.3899 \times 10^{-}$ ${ }^{12}$ to $-6.3908 \times 10^{-12} \mathrm{~Pa}^{-1}$, with a mean of $-6.3902 \times$ $10^{-12} \mathrm{~Pa}^{-1}$ and a relative $95 \%$ confidence interval ( $\left.2 \sigma_{\bar{x}}\right)$ of $\pm 3 \times 10^{-16} \mathrm{~Pa}^{-1}$, corresponding to $\pm 50 \mathrm{ppm}$ (parts-per-million). Since the simulations were performed under slightly different conditions, with somewhat dissimilar procedures, and this confidence interval corresponds, for $\mathrm{N}_{2}$, to a relative uncertainty in the assessed refractivity of $0.1 \mathrm{ppm}$, this was found adequate. The results are, for clarity, summarized in Table 3.
Table 3. Summary of the simulated pressurenormalized cavity deformation using the finest meshing by each partner and software.

\begin{tabular}{|l|c|}
\hline Partner - Software used & $\begin{array}{c}\text { Pressure-normalized } \\
\text { deformation: } \\
(\Delta L / L) / P \\
\text { in } \mathbf{1 0}^{-\mathbf{1 2}} \mathbf{P a}^{-\mathbf{1}}\end{array}$ \\
\hline $\mathbf{A}-$ Comsol & -6.3900 \\
\hline $\mathbf{B}-$ Comsol & -6.3899 \\
\hline $\mathbf{B}-$ Ansys & -6.3908 \\
\hline $\mathbf{C}-$ Ansys & -6.3900 \\
\hline $\mathbf{D}-$ Comsol & -6.3903 \\
\hline \hline Average value & -6.3902 \\
\hline $\begin{array}{l}\text { Standard error of the } \\
\text { mean }\left(\sigma_{\bar{x}}\right)\end{array}$ & $1.5 \times 10^{-4}$ \\
\hline $\begin{array}{l}\text { 95\% }\left(2 \sigma_{\bar{x}}\right) \text { confidence } \\
\text { interval }\end{array}$ & $2.9 \times 10^{-4}$ \\
\hline
\end{tabular}

\section{DISCUSSION}

\subsection{Technicalities}

It can be noticed that the simulations performed on the entire cavity (by partner A) in virtually all cases predicted a deformation that is smaller than those addressing $1 / 8$ of the cavity. However, when the entire cavity is simulated, there are roughly eight times more mesh elements than what would be if $1 / 8$ of the cavity would be simulated. By recalculating the numbers of mesh elements used in the simulations of $1 / 8$ of the cavity to those for the entire cavity, the results of the $1 / 8$ of the cavity conform to the simulations of the entire cavity. From this we conclude that using symmetry planes can, for this cavity, be seen as appropriate.

Similarly, although there is a tendency that the simulations made using the ANSYS Workbench software (by partner B and C) consistently predict larger deformations than those made on the COMSOL Multiphysics ${ }^{\circledR}$ software (for comparable number of mesh elements), the difference in simulated deformation performed by the two types of software when the largest numbers of mesh points were used (partner B, Ansys, $2.9 \times 10^{6}$ elements, and partner D, Comsol, $3.2 \times 10^{6}$ elements, respectively), is only $5 \times 10^{-16} \mathrm{~Pa}^{-1}$, which, for $\mathrm{N}_{2}$, corresponds to a relative uncertainty in the assessed refractivity of solely $0.17 \mathrm{ppm}$. This indicates that when a large number of mesh elements are being used, the difference between the two types of software is insignificant in comparison to several other types of uncertainties, e.g., those from dynamic molar polarizabilities.

It is also possible that some other meshing parameters (or settings) than those identified in this report can affect the simulations. However, no study of such phenomena was addressed in this comparison. 


\subsection{Consensus}

As was alluded to above, the simulations performed by the various partners, utilizing dissimilar software and set-up strategies, did finally, for a given geometry and set of material parameters, provide a relative $95 \% \quad\left(2 \sigma_{\bar{x}}\right)$ confidence interval of $\pm 3 \times 10^{-16} \mathrm{~Pa}^{-1}$. For the FP cavity addressed, this corresponds, for $\mathrm{N}_{2}$, to a relative uncertainty in the assessed refractivity solely of $0.1 \mathrm{ppm}$, which, for the pertinent QuantumPascal EMPIR project, is considered adequate.

\subsection{Accuracy}

The deformation of this cavity has previously been experimentally assessed to $(6.030 \pm 0.005) \times$ $10^{-12} \mathrm{~Pa}^{-1}[8]$. The simulated values differ systematically $6 \%$ from this. Since the accuracy of the simulations is given by the accuracy of both the cavity spacer material parameters and the geometrical quantities, and none of these where considered having any uncertainty in this work (see e.g. Table 1), it is possible that this discrepancy can, to a certain extent, be explained by uncertainties in these entities.

It is also possible that the accuracy is affected by features not included in the simulations, e.g. the mounting of the cavity, gravity, or aging. Although such effects were not a part of the A1.1.1 task in the 18SIB04 QuantumPascal EMPIR project under which this study was performed, the influence of the former of these was investigated by one of the partners (A) who showed that the mounting of the cavity indeed could affect the final deformation.

It is also plausible that the deviations between the simulated and the experimentally assessed values can be attributed to yet unidentified experimental processes or features.

It is finally worth to note that for cavities with more complex design (e.g. with more intricate mirror mounting), the accuracy is likely to be adversely affected by the fact that the actual cavity geometry will be less accurately known.

\section{SUMMARY AND CONCLUSION}

The ability of four partners of the 18SIB04 QuantumPascal EMPIR project to simulate, by FEM-simulations, the pressure-induced cavity deformation of a given FP cavity has been assessed. When setting up the simulations, it was found that considerable care must be taken regarding the input parameters and boundary conditions since any minor inaccuracies in these could lead to non-negligible errors in the simulations.

In the absence of methodological inconsistencies, and for a given geometry and set of material parameters, adequate agreement between the simulations made by the various partners was reached. It was found that the use of different types of software did not significantly affect the results. On the other hand, a certain dependence on the number of mesh elements used was found. It was found that the remaining discrepancies were small, with a $2 \sigma$ confidence interval solely of $\pm 50 \mathrm{ppm}$. Since this spread in the predicted cavity deformation corresponds to an uncertainty in assessed refractivity of $\mathrm{N}_{2}$ and He solely of 0.1 and 0.9 ppm, respectively, this was considered adequate for the project.

Although good consensus among the various partners regarding simulated pressure-induced strain of the cavity considered was reached, a comparison with a previous experimentally assessed deformation assessment indicated a $6 \%$ deviation. Considering that the most probable values of all material parameter were used in the simulations, this indicates that it may be unlikely that simulations by themselves can provide lowppm accuracy in assessment of refractivity. However, since accuracy and compliance with experimental data were not an issue of the A1.1.1 activity under which this work was performed, no further investigations of the causes for the observed discrepancy between simulated and experimental pressure-induced strain were performed; it was considered sufficient to demonstrate consensus regarding set up and execution of the FEM software used for the simulations. Instead, the upcoming activities in the project, in which each partner will model the deformation of a FPC they have access to in their own laboratory whose deformation they also will experimentally characterize, will assess to which extent simulations are a feasible means to assess cavity deformation.

This project (QuantumPascal, 18SIB04) has received funding from the EMPIR programme cofinanced by the Participating States and from the European Union's Horizon 2020 research and innovation programme; the Swedish Research Council (VR) (Project No. 621-2015-04374); the Umeå University Industrial Doctoral School (IDS); and the Kempe Foundations (Project No. 1823, U12).

\section{REFERENCES}

[1] C.R. Tilford, "3 and a half centuries later - the modern-art of liquid-column manometry", Metrologia, vol. 30, pp. 545-552, 1994.

[2] S. Semenoja and M. Rantanen, "Comparisons to establish a force-balanced piston gauge and a spinning rotor gauge as the new measurement standards of MIKES", Vacuum, vol. 73, pp. 269274, 2004.

[3] J. H. Hendricks, D. A. Olson, “1-15,000 Pa Absolute mode comparisons between the NIST 
ultrasonic interferometer manometers and nonrotating force-balanced piston gauges, Measurement, vol. 43, pp. 664-674, 2010.

[4] J. Ricker, J. Hendricks, T. Bock, P. Dominik, T. Kobata, J. Torres, I. Sadkovskaya, "Final report on the key comparison CCM.P-K4.2012 in absolute pressure from $1 \mathrm{~Pa}$ to $10 \mathrm{kPa}$, Metrologia, vol. 54, p. 07002, 2017.

[5] Physikalisch-Technische Bundesanstalt (PTB), 18SIB04 QuantumPascal, https://www.ptb.de/empir2019/quantumpascal/ho me/ , (accessed: 18.09.2020).

[6] M. Stock, R. Davis, E. de Mirandés, and M. J. T. Milton, "The revision of the SI-the result of three decades of progress in metrology", Metrologia, vol. 56, pp. 049502, 2019.

[7] T. Rubin, Y. Yang, "Simulation of pressure induced length change of an optical cavity used for optical pressure standard", Journal of Physics: Conf. Series, vol. 1065, p. 162003, 2018.

[8] Z. Silvestri, F. Boineau, P. Otal and J. Wallerand, "Helium-Based Refractometry for Pressure Measurements in the Range 1-100 kPa," in IEEE (ed.), 2018 Conference on Precision Electromagnetic Measurements, (IEEE, New York, NY 10017, USA, 2018). 